\title{
Gérard NOIRIEL, Dire la vérité au pouvoir. Les
} intellectuels en question

Nouvelle éd. revue et actualisée, Paris, Agone, coll. Éléments, 2010, 316 p.

Jean Zoungrana

\section{CpenEdition}

\section{Journals}

Édition électronique

URL : https://journals.openedition.org/questionsdecommunication/6755

DOI : 10.4000/questionsdecommunication.6755

ISSN : 2259-8901

Éditeur

Presses universitaires de Lorraine

Édition imprimée

Date de publication : 1 septembre 2012

Pagination : 319-322

ISBN : 978-2-8143-0120-7

ISSN : 1633-5961

Référence électronique

Jean Zoungrana, "Gérard NoIRIEL, Dire la vérité au pouvoir. Les intellectuels en question », Questions de communication [En ligne], 21 | 2012, mis en ligne le 18 décembre 2012, consulté le 27 octobre 2022 URL : http://journals.openedition.org/questionsdecommunication/6755 ; DOI : https://doi.org/ 10.4000/questionsdecommunication.6755

\section{cc) (i) $\Theta$}

Creative Commons - Attribution - Pas d'Utilisation Commerciale - Pas de Modification 4.0 International - CC BY-NC-ND 4.0

https://creativecommons.org/licenses/by-nc-nd/4.0/ 
entre 1793 et 1855 , a profondément bouleversé les pratiques et les habitudes des français. En outre, « la nouvelle temporalité de l'univers ferroviaire a été le point de départ d'une nouvelle temporalité tout court » (p. 69). C'est avec l'heure ferroviaire que s'est déclenché le processus d'harmonisation qui a mené avant la fin du XIX siècle à l'émergence d'une heure mondiale, acceptée et utilisée par tous. Les progrès de la technique à la fin du XIXe siècle ont présidé à l'invention de la figure du réseau de communication perçu comme créateur de nouveau lien universel.

Saint-Simon, le premier, considère la société comme un organisme vivant « considéré comme un enchevêtrement ou un tissage de réseaux » (p. 102). La création de l'Alliance française en 1883 répond à un besoin de propagation de la langue française dans les colonies et à l'étranger. C'est ce qui fait écrire à Armand Mattelart que « le langage de la propagation et, au-delà, le modèle religieux de la propagande imprègnent les modes de dire et de faire la communication »(p.209). Conçue comme une association privée (mais agissant en bonne intelligence avec les ministères de l'Instruction publique et des Affaires étrangères), l'Alliance française s'appuie sur de nombreux réseaux de communication de par le monde et « sur les circuits d'influence qu'ont tissé et continuent à tisser ses adhérents : les écoles publiques françaises à l'étranger, les établissements d'enseignement des missions protestantes et catholiques, les écoles de l'Alliance israélite ouvertes sur tous les points du globe » (p. 2 I I). En 1948, Claude Shannon formule la première théorie mathématique de l'information et de la communication pour le compte des laboratoires de la compagnie téléphonique Bell : «Dès ses débuts, la théorie mathématique de l'information s'installe comme passe-muraille des disciplines et irrigue, grâce à sa puissance d'organisation, des champs de connaissance aussi divers que l'économie ou la physique, la sociologie, la psychologie ou la linguistique » (p. 338). À la fin du XIXe siècle, la communication appelait de ses vœux la solidarité universelle et l'interdépendance biologique dans un monde certes menacé par la guerre, mais où l'on croyait à la redistribution sociale et à la résorption des inégalités. Juste après la fin de la Seconde Guerre mondiale, le média devient un aiguillon capable de déclencher le changement social. Armand Mattelart observe avec beaucoup de subtilité que l'une des fonctions essentielles des dispositifs de communication électronique consiste à protéger l'usager des dispositifs de communication de la violence de l'autre, véritable laissé-pour-compte des réseaux : «Plus tombent les entraves au free flow des marchandises et à la libre circulation de ses officiants, plus les grands groupes multimédias et multinationaux font de la surenchère sur leur vocation passe-frontières, plus aussi s'instaure la pratique du passeport électronique pour les "excommuniés" » (pp.34l-342). Dans l'épilogue de son ouvrage, l'auteur plaide pour la réappropriation par l'homme de ce monde « machinique » omniprésent de nos jours. Cette réappropriation est « d'autant plus cruciale que la "communication" est en passe de devenir dans nos sociétés un objet fantasmatique et fantasmagorique sur le dos duquel spéculent démagogues et démiurges. Raison de plus pour la faire échapper à cet univers amnésique en réinsufflant un peu d'histoire afin de l'imaginer autrement » (p. 345). Son essai constitue une invitation à repenser la communication dans la diachronie et de ne pas sacrifier à la mode de l'immédiat et de l'ultra-récent. En tant que passeur d'une mémoire des théories et des savoirs sur la communication, Armand Mattelart s'impose comme un remarquable pédagogue ayant mené une recherche de grande ampleur. Enjeu de société et vecteur de changement, la communication s'impose comme un bon moyen pour bouleverser les mentalités et les habitus culturels, comme l'écrit l'auteur dans la postface inédite à son ouvrage. La révolution des communications annoncée dans les années 70 (et sous-tendue par un continu savoirpouvoir) n'en est qu'à ses balbutiements. Nul doute qu'à l'avenir, « la construction de la démocratie par le partage et l'échange des savoirs n'empruntera pas les autoroutes mais les petites routes de campagne et les chemins de traverse » (p. 386). Gageons que le lecteur se laissera guider par sa curiosité pour emprunter ces routes et ces chemins de la communication.

\section{Alexandre Eyries \\ 13M, université de Nice Sophia Antipolis alex.eyries@yahoo.fr}

\section{Gérard NoIRIEL,Dire la vérité au pouvoir. Les intellectuels en question.}

Nouvelle éd. revue et actualisée, Paris, Agone, coll. Éléments, 2010,316 p.

Ce livre est une interrogation sur le rôle et la place des intellectuels dans la cité. Pour ce faire, Gérard Noiriel prend appui sur une longue enquête menée auprès de figures emblématiques d'intellectuels qu'ils cherchent à décrypter telles que Charles Péguy, Paul Nizan, Jean-Paul Sartre, Michel Foucault, Pierre Bourdieu, François Furet, Jacques Rancière. Deux principes semblent se confronter tout au long cette analyse : le principe d'autonomie du savant à l'égard 
du politique et celui du lien entre savant et politique. Comment s'articulent la théorie et la pratique dans l'ordre social et politique, telle est la question qui traverse de part en part ce texte.

Mais il convient d'entrée de jeu de relever que cette dernière livraison est en fait une version fortement remaniée de son précédent ouvrage paru en 2005 sous le titre Les Fils maudits de la République. L'avenir des intellectuels en France (Paris, Fayard). C'est ainsi que dès l'entame de son livre, l'auteur s'explique sur les raisons qui l'ont amené à cette réécriture. Plusieurs raisons donc. La première, c'est la nécessité d'un retour aux fils maudits de la République, au regard de la situation actuelle, sur la posture de l'intellectuel spécifique incarnée par Pierre Bourdieu lors de la grève des cheminots en novembre/décembre 1995 La seconde raison, plus fondamentale à ses yeux, imposait de revenir sur le contenu même du livre à remanier afin de ne pas donner à voir, à propos de ce livre, une simple version d'une histoire des intellectuels. Car, il s'agissait bien pour lui de questionner le type de connaissances que mobilisent les intellectuels dans leurs interventions dans la cité. Enfin, quel sens donner à la notion d'intellectuel critique ? Telle est la troisième raison qui a prévalu à l'écriture de ce nouvel essai qui se veut un approfondissement du premier. Les réponses apportées au prix d'une abondante documentation sont à la hauteur du questionnement : riches et éclairantes, pour qui s'intéresse à cette thématique.

En choisissant de privilégier le prototype des universitaires dans la définition de l'intellectuel, il parvient à mesurer et analyser les tensions à l'œuvre « entre deux types d'aspirations contradictoires, à savoir le désir de vérité et le souci de l'action » (p.8) Ce sont bien ces tensions qui sont en jeu dans la distinction entre le savant et le politique. Et l'affaire Dreyfus va jouer un rôle essentiel dans le surgissement de cette figure de l'intellectuel. C'est aussi l'occasion pour Gérard Noiriel de mettre en avant le contexte sociopolitique, dominé par la question sociale, dans lequel éclate l'affaire du capitaine Dreyfus, juif et alsacien : professionnalisation de la politique, émergence du journaliste après celle du publiciste, place des enseignants, chercheurs et autres experts dans la société, instauration de la démocratie républicaine portée par la Troisième République. En tout cas, si les intellectuels tirent leur existence du vide créé par la séparation entre le savant et le politique opéré au XIXe siècle en France, l'affaire Dreyfus, par l'émotion suscitée et l'injustice ressentie, sera l'occasion pour eux d'occuper l'espace public et ainsi contribuer à forger l'usage et l'extension du terme intellectuel : au fond, ils réussissent à retourner à leur avantage cette désignation d'intellectuel qui, à l'origine, était utilisée par les antidreyfusards pour précisément les disqualifier en les stigmatisant. Ce faisant, ils parviennent à maintenir le lien entre le savant et le politique car, c'est bien comme savants que ces universitaires prennent position publiquement pour Dreyfus, contrairement au principe républicain de la séparation du savant et du politique. Mais aussi et surtout, cette issue victorieuse de leur combat les conforte dans leur position affirmée du lien entre savant et politique.

Comment maintenir le lien entre savant et politique au-delà de l'effervescence d'un combat ? Telle est la question, restée à jamais ouverte, qui va continuer de tarauder nombre d'intellectuels. Si donc l'intellectuel se présente comme celui qui « dit la vérité au pouvoir au nom des opprimés », trois profils d'intellectuels s'imposent à l'analyse : l'intellectuel révolutionnaire qui s'installe dans une critique $d u$ pouvoir, l'intellectuel de gouvernement qui tend à orienter l'opinion, l'intellectuel spécifique qui cherche à éclairer les citoyens. C'est autour de ces trois figures emblématiques que Gérard Noiriel choisit d'organiser son argumentation.

Dans la première catégorie d'intellectuels, celle des intellectuels révolutionnaires, Gérard Noiriel privilégie deux figures, Georges Sorel et Charles Péguy, tous deux ayant découvert l'action politique à travers leur participation aux luttes sociales dans les années 1890. En effet, le rôle de ces deux personnalités fut de dénoncer les solutions proposées par les intellectuels de gouvernement, c'est-à-dire ceux qui avaient choisi de se mettre au service du pouvoir. Leur impératif à valeur de slogan était le suivant : «Dire la vérité au pouvoir, au nom des opprimés ». Pour Charles Péguy, après avoir refusé une carrière d'enseignant, le lien entre le savant et le politique se fait par l'intermédiaire de la littérature. Se voulant proche des opprimés, il se présente comme leur porte-parole. Quant à Georges Sorel, c'est à travers sa compétence scientifique (ingénieur de formation) qu'il parvient à maintenir le lien entre le savant et le politique. Si Karl Marx est le prototype de l'universitaire qui, au nom de la cause des opprimés, a su allier œuvre théorique (posture de savant) et action pratique (posture politique), Georges Sorel verra en lui un modèle incontournable à imiter. C'est ce qui le conduira à diffuser le marxisme en France à la fin du XIX ${ }^{\text {e }}$ siècle et à en faire une arme de destruction contre l'idéologie dominante. 
Pour Georges Sorel comme pour Charles Péguy, le souci de l'action n'était pas une simple fiction; ils l'ont vraiment vécu dans leur chair.Tous les deux ont choisi de vivre la condition du grand nombre, c'est-à-dire dans la pauvreté : «Sorel a renoncé à la retraite à laquelle il avait droit et Péguy a vécu dans une situation proche de celle du prolétariat intellectuel de son temps 》 (p. 83)

La deuxième génération d'intellectuels, à savoir celle d'intellectuels révolutionnaires est représentée par Paul Nizan et Jean-Paul Sartre. Comme pour résoudre le problème de la séparation du savant et du politique, tous les deux ont rompu avec leur corps d'origine (la fonction publique) et leur milieu social (bourgeois). Jean-Paul Sartre encore plus. Paul Nizan le présente comme «l'homme qui dit non jusqu'au bout » (p. 92) : refus de la Légion d'honneur et du prix Nobel de littérature. Toutefois, reconnu à la fois comme philosophe, écrivain et acteur majeur de la vie politique, Jean-Paul Sartre, à travers tout le témoignage de sa vie, a réussi à proposer de fait une solution au problème de la séparation du savant et du politique au tournant des années 70. La figure de l'intellectuel s'impose alors dans l'espace public. Mais pas pour longtemps.

Car très vite, à ce modèle sartrien, succèdera une autre figure, celle de l'intellectuel critique symbolisé par Michel Foucault, Jacques Derrida, Gilles Deleuze qui vont eux aussi réussir à combler, à leur façon, le fossé entre le savant et le politique. Toutefois, à ce niveau d'analyse, Gérard Noiriel choisit de s'intéresser davantage à l'œurre critique de Jacques Rancière, notamment à ses questionnements sur les sciences sociales. En effet, celui-ci, tirant leçons de sa propre posture de surplomb, n'aura de cesse de soumettre à la critique toute posture de domination, y compris celle des intellectuels en vue comme Pierre Bourdieu et naguère Louis Althusser.

Et qu'en est-il des historiens au-delà de cette philosophie critique? Pour eux, il s'agit, note l'auteur, d'arriver à établir une connexion féconde entre la recherche et l'actualité pour être perçu comme intellectuel. Cette démarche initiée par Albert Mathiez ressemble aujourd'hui à celle entreprise par Pap Ndiaye dans son ouvrage sur La condition noire: essai sur une minorité française (Calmann-Lévy, 2008) : ouvrir, en historien critique, un chantier de recherches savantes sur la « question noire » qui semble désertée par les sciences sociales en France.
Pour montrer ce qu'est le rôle des intellectuels de gouvernement, Gérard Noiriel emprunte une formule de Guizot opportunément rappelée par Pierre Rosanvallon : " Gouverner par le maniement des esprits et non par le bouleversement des existences $\gg$ (p. 137). C'est l'historien qui fera figure d'intellectuel de gouvernement en réussissant à imposer l'histoire comme moyen d'éducation politique. À l'origine de cette émergence, trois figures et hommes de réseaux: Charles Seignobos qui jouera un rôle décisif dans la réforme du système universitaire français, dans la définition des programmes et dans l'édition ;André Siegfried, professeur au Collège de France, qui pèsera de tout son poids sur l'édition des manuels ; Alfred Fouillée, très influent dans les réseaux républicains.

Bien plus. D'autres figures contemporaines vont consolider cette emprise des historiens dans l'ordre gouvernemental : René Rémond et François Furet qui, dès l'entame de leur vie professionnelle, ont mené de front des activités savantes et politiques. Et ce qu'observe Gérard Noiriel, c'est la forte présence de la politique dans l'ascendance familiale de ces historiens de gouvernements qui se pensent à la fois comme hommes de sciences et hommes d'action. Leur position au sein d'institutions de référence contribuera à asseoir leur notoriété et légitimité auprès d'un large public et des lieux de pouvoir. René Rémond a présidé aux destinées de l'université de Paris 10-Nanterre (197| | 1976), la Fondation nationale de sciences politiques (depuis |981),I'Institut d'histoire du temps présent (1979-1990). François Furet, conseiller d'Edgar Faure lors de la réforme de l'enseignement supérieur en 1968, en tirera une riche expérience pour présider l'École des hautes études en sciences sociales (EHESS) et surtout orienter la recherche vers I'histoire contemporaine. Bénéficiant aussi d'une audience internationale (professeur à l'université de Chicago), il aura également un rayonnement sur la société civile (président de la Fondation Saint-Simon). Et, comme pour boucler la boucle, tous deux seront élus à l'Académie française, reçus dans le cercle des Immortels.

Mais, paradoxalement, la réussite des intellectuels de gouvernement est aussi la principale cause de leur crise. En effet, comme le souligne Gérard Noiriel « l'intellectuel et l'académicien n'ont jamais fait bon ménage, car il est difficile, quand on appartient à l'élite de l'élite, de se présenter comme un porte-parole des opprimés » (p. 189). Cette crise sera plus manifeste lors de la grève des cheminots en novembredécembre 1995. En prétendant dire la vérité aux opprimés au nom du pouvoir, les intellectuels de 
gouvernement ont perdu tout crédit « intellectuel » que leur conféraient leurs positions dominantes dans les institutions : l'intellectuel étant d'abord et avant tout, comme le rappelle Gérard Noiriel, celui qui dit la vérité au pouvoir au nom des opprimés. De plus, la critique portée par les intellectuels critiques et les intellectuels spécifiques contribuera à entamer leur audience et leur crédit.

Dans ces conditions, quelle est l'originalité des intellectuels spécifiques? II faut d'abord noter que l'expression « intellectuel spécifique » date des années 70 ; son rôle a été ainsi défini par Michel Foucault, comme le rappelle Gérard Noiriel : « Le travail d'un intellectuel n'est pas de modeler la volonté politique des autres. II est, par les analyses qu'il fait dans les domaines qui sont les siens, de réinterroger les évidences et les postulats, de secouer les habitudes, les manières de faire et de penser » (p. 209). En partant de ce constat avec un regard rétrospectif, on peut dire qu'au fond, le premier intellectuel spécifique avant le concept a été Emile Durkheim. En effet, toute son œuvre peut se lire comme un effort pour échapper au déterminisme de la race très présent à l'époque dans les sciences sociales. C'est pourquoi dans son intervention sur l'affaire Dreyfus, il reste cantonné dans son domaine de compétence sans se prononcer sur l'affaire elle-même. Cette même posture d'intellectuel spécifique se retrouve chez Claude Lévi-Strauss qui a été directeur de l'École (pratique), professeur au Collège de France et membre de l'Académie française : il n'aura de cesse de défendre l'autonomie de la science à l'égard du politique.

Mais les deux intellectuels spécifiques à avoir marqué les dernières décennies du $X X^{e}$ siècle sont Michel Foucault et Pierre Bourdieu. Toutefois, il faut noter que c'est avec Michel Foucault que cette posture de l'intellectuel spécifique se trouve la plus affirmée même si, au fil des analyses, il oscille entre la posture de l'intellectuel critique et celle de l'intellectuel spécifique. Lui aussi est conduit à prôner l'autonomie de la science de sorte que son œuvre apparaît comme une série d'études spécifiques sur des thèmes d'époque (la folie, la prison, la sexualité, etc.) à l'usage des dominés. Chez Pierre Bourdieu, on note un souci d'être utile aux autres qui le conduit à osciller entre une posture d'intellectuel spécifique et une posture d'intellectuel critique pour finalement adopter celle du savant-écrivain désireux de faire partager son expérience telle que proposée par Michel Foucault dans les années 70 : « Et rien ne me rendrait plus heureux que d'avoir réussi à faire que certains de mes lecteurs/lectrices reconnaissent leurs expériences, leurs difficultés, leurs interrogations, leurs souffrances, etc., dans les miennes et qu'ils tirent de cette identification réaliste, ce qui est tout à fait à l'opposé d'une projection exaltée, des moyens de faire et de vivre un tout petit mieux ce qu'lls vivent et ce qu'ils font » (p. 258). Ce retour à soi ou sur soi sonne comme une posture d'authenticité qui, seule, peut parler dans le vrai. II reste qu'aucun de ces intellectuels ne semble pleinement satisfait de son positionnement. On pourrait donc se demander si l'insatisfaction n'est pas ce qui, précisément, définit l'intellectuel (universitaire), voire ce qui l'habite et le hante et, par conséquent, le jette dans une quête inlassable de positionnement, tant la question du savant et du politique nécessite un questionnement continu.

Avec ce livre, Gérard Noiriel offre une plongée dans la vie intellectuelle française depuis l'affaire Dreyfus jusqu'à nos jours. Loin d'être simplement linéaire, l'exposé opère des va-et-vient d'une époque à l'autre ; ce qui permet d'éclairer autrement les enjeux à l'œuvre et les tensions souterraines. Son originalité et sa méthode résident dans la convocation de l'histoire personnelle de chacun des protagonistes. En effet, l'histoire personnelle n'est jamais étrangère à la trajectoire de ces intellectuels. Des éléments biographiques, voire autobiographiques, permettent d'éclairer certaines orientations professionnelles ou options théoriques: des difficultés de socialisation éprouvées dans l'enfance communes aux intellectuels révolutionnaires et aux intellectuels spécifiques aux facteurs sociaux déterminants pour les intellectuels de gouvernement. On note bien dans cet ouvrage de Gérard Noiriel des linéaments pour une sociogenèse des intellectuels français.

Jean Zoungrana

CRESS, université de Strasbourg zoungrana@unistra.fr

loana PoPA, Traduire sous contraintes. Littérature et communisme (1947-1989).

Paris, cnRs Éd., coll. Culture et société, 2010,590 p.

« Rideau de fer 》, « mur 》, « frontière infranchissable » (p. 5), ces métaphores sont liées à la connaissance politique des régimes communistes en Europe de l'Est avant la chute du mur de Berlin. Avec l'application du modèle soviétique aux pays d'Europe de l'Est après la Seconde Guerre mondiale, les conditions de publication et d'exercice du métier d'écrivain ont été transformées par suite du contrôle idéologique et de l'installation d'une censure « préventive et 